\title{
EXISTENCE, UNIQUENESS, AND VARIATIONAL METHODS FOR SCATTERING BY UNBOUNDED ROUGH SURFACES*
}

\author{
SIMON N. CHANDLER-WILDE ${ }^{\dagger}$ AND PETER MONK ${ }^{\ddagger}$
}

\begin{abstract}
In this paper we study, via variational methods, the problem of scattering of time harmonic acoustic waves by an unbounded sound soft surface. The boundary $\partial D$ is assumed to lie within a finite distance of a flat plane and the incident wave is that arising from an inhomogeneous term in the Helmholtz equation whose support lies within some finite distance of the boundary $\partial D$. Via analysis of an equivalent variational formulation, we provide the first proof of existence of a unique solution to a three-dimensional rough surface scattering problem for an arbitrary wave number. Our method of analysis does not require any smoothness of the boundary which can, for example, be the graph of an arbitrary bounded continuous function. An attractive feature is that all constants in a priori bounds, for example the inf-sup constant of the sesquilinear form, are bounded by explicit functions of the wave number and the maximum surface elevation.
\end{abstract}

Key words. nonsmooth boundary, radiation condition, a priori estimate, inf-sup constant, Helmholtz equation

AMS subject classifications. 35J05, 35J20, 35J25, 42B10, 78A45

DOI. $10.1137 / 040615523$

1. Introduction. This paper is concerned with the development and analysis of a variational formulation for scattering by unbounded surfaces, in particular, with the study of what are termed rough surface scattering problems in the engineering literature. We shall use the phrase rough surface to denote surfaces which are a (usually nonlocal) perturbation of an infinite plane surface such that the whole surface lies within a finite distance of the original plane. Such problems arise frequently in applications, for example in modeling acoustic and electromagnetic wave propagation over outdoor ground and sea surfaces, and are the subject of intensive studies in the engineering literature, with a view to developing both rigorous methods of computation and approximate, asymptotic, or statistical methods (see, e.g., the reviews and monographs by Ogilvy [23], Voronovich [26], Saillard and Sentenac [24], Warnick and Chew [27], and de Santo [13]).

In this paper we will focus on a particular, typical problem of the class, which models time harmonic acoustic scattering by a sound soft rough surface. In particular, we seek to solve the Helmholtz equation with wave number $k>0, \Delta u+k^{2} u=g$, in the perturbed half-plane or half-space $D \subset \mathbb{R}^{n}, n=2,3$. We suppose that the homogeneous Dirichlet boundary condition $u=0$ holds on $\partial D$, and a suitable radiation condition is imposed to select a unique solution to this problem. We shall give in the next section complete details about our assumptions on $D$ and on the radiation condition, but we now note that the inhomogeneous term $g$ might be in $L^{2}(D)$ with bounded support, or be a more general distribution. In addition the boundary $\partial D$ may or may not be the graph of a function.

${ }^{*}$ Received by the editors September 22, 2004; accepted for publication (in revised form) February 1, 2005; published electronically October 27, 2005. This work was supported by the UK Engineering and Physical Sciences Research Council under grant GR/S67401.

http://www.siam.org/journals/sima/37-2/61552.html

${ }^{\dagger}$ Department of Mathematics, University of Reading, Whiteknights, PO Box 220, Berkshire RG6 6AX, UK (S.N.Chandler-Wilde@reading.ac.uk).

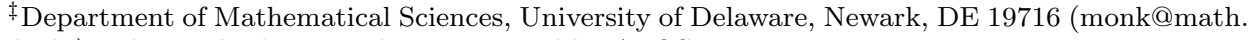
udel.edu). This author's research is supported by AFOSR grant F49620-02-1-0071. 
The main results of the paper are the following. In the next section we formulate the boundary value problem precisely, in the case when $g \in L^{2}(D)$ with support lying within a finite distance of $\partial D$. We also establish the equivalent variational formulation that we use and study in this paper. As part of the boundary value problem formulation we require the radiation condition often used in a formal manner in the rough surface scattering literature (e.g., [13]) that, above the rough surface and the support of $g$, the solution can be represented in integral form as a superposition of upward traveling and evanescent plane waves. This radiation condition is equivalent to the upward propagating radiation condition proposed for two-dimensional (2D) rough surface scattering problems in [11], and has recently been analyzed carefully in the 2D case by Arens and Hohage [5]. Arens and Hohage also propose a further equivalent radiation condition (a "pole condition").

In section 3 we analyze the variational formulation in the long wavelength case, showing that the sesquilinear form is then elliptic, so that unique existence of solution and explicit bounds on the solution in terms of the data $g$ follow from the Lax-Milgram lemma.

In section 4 we show that, for an arbitrary wave number $k$, the variational problem and the equivalent boundary value problem remain well-posed in the case when the rough surface has the property that if $x$ lies in $D$, then every point above $x$ lies in $D$. Our methods of argument, which depend on an a priori estimate established via a Rellich-type identity, application of the generalized Lax-Milgram theory of Babuška, and results on approximation of nonsmooth by smooth domains, lead to simple, explicit lower bounds on the inf-sup constant of the sesquilinear form and corresponding explicit bounds on the solution in terms of the data $g$. We note that, in contrast to earlier uniqueness and existence results for rough surface scattering problems, no additional regularity conditions on the boundary are required; our theorem applies, for example, whenever the boundary $\partial D$ is the graph of a bounded continuous function.

The results and methods of our paper are closest to those of Kirsch [20] and Elschner [16]. These authors study the same problem tackled in this paper, but consider the 2D diffraction grating case when $\partial D=\left\{\left(x_{1}, f\left(x_{1}\right)\right): x_{1} \in \mathbb{R}\right\}$ with $f$ periodic and $g$ quasi-periodic (i.e., $g(x) \mathrm{e}^{\mathrm{i} \alpha x_{1}}$ is periodic with the same period as $f$ for some $\alpha \in \mathbb{R})$. The variational formulation we propose for the rough surface scattering problem is analogous to that considered for the periodic case in $[20,16]$. We note, however, that the periodicity simplifies the mathematical arguments considerably compared to the case we study; the variational formulation is over a bounded region, part of a single period of the domain, so that compact embedding arguments can be applied and the sesquilinear form which arises satisfies a Gårding inequality for all wave numbers. We note, moreover, that the methods of $[20,16]$ require $f$ to be at least Lipschitz continuous, and do not lead to explicit bounds on stability constants.

The methods of argument used to prove uniqueness in $[20,16]$ derive, in part, from Alber [2] and Cadilhac [7]. In fact the argument outlined in [7] for the 2D diffraction grating problem could be adapted to prove uniqueness of solution for our boundary value problem in the case when $\partial D$ is the graph of a sufficiently smooth function. However, we will prefer to establish uniqueness via an a priori bound which also leads to an existence result.

In the general 2D case when $f$ is not periodic, existence of a unique solution to the boundary value problem we study has been established via integral equation methods in the case that $f \in C^{2}(\mathbb{R})\left(\partial D\right.$ is $\left.C^{2}\right)$, and well-posedness of the integral equation formulation has been established in a variety of function spaces [11, 10, 9, 3, 4]. The 
extension to the case when $\partial D$ is Lipschitz is outlined in Zhang [28]. To date, however, the only existence result [8] for the three-dimensional rough surface problem, derived via integral equation methods, applies only to the Dirichlet boundary value problem for the Helmholtz equation when the rough surface is the graph of a sufficiently smooth function with sufficiently small surface slope, and deals only with the case when the wave number is sufficiently small.

In another, somewhat related body of work existence of solution to the Dirichlet problem for the Helmholtz equation, with $\partial D$ unbounded, is established by the limiting absorption method, via a priori estimates in weighted Sobolev spaces (see Eidus and Vinnik [14], Vogelsang [25], Minskii [22], and references therein). The results obtained apply to the problem considered in this paper, but only if we assume that the rough surface approaches a flat boundary sufficiently rapidly at infinity and/or that the sign of $x \cdot \nu(x)$ is constant on $\partial D$ outside a large sphere, where $\nu(x)$ denotes the unit normal at $x \in \partial D$. Moreover, this body of work requires that $g$ decrease sufficiently rapidly at infinity so that a Rellich-Sommerfeld radiation condition is satisfied.

An attractive feature of our results is the explicit bounds we obtain on the solution in terms of the data $g$, which exhibit explicitly dependence of constants on the wave number and on the geometry of the domain. In part our methods of argument to obtain our bounds are inspired by the work of Melenk [21] and by the closely related work of Cummings and Feng [12]. In these publications bounds, exhibiting explicit dependence on the wave number, are developed for the impedance boundary value problem for the Helmholtz equation in a bounded domain which is either convex or smooth and star-like.

In this paper we propose a variational formulation and exploit it as a theoretical tool to study the well-posedness of the boundary value problem. We anticipate that the variational formulation will also be very suitable for numerical solution via finite element discretization, as are similar formulations for the 2D diffraction grating case $[6,15,16]$. Moreover, the explicit bounds we obtain should be helpful in establishing the dependence, on the wave number and the domain, of the constants in a priori error estimates for finite element schemes. These numerical analysis aspects will be considered in a future paper.

2. The boundary value problem and variational formulation. In this section we shall define some notation related to the rough surface scattering problem and write down the boundary value problem and equivalent variational formulation that will be analyzed in later sections. For $x=\left(x_{1}, \ldots, x_{n}\right) \in \mathbb{R}^{n}(n=2,3)$ let $\tilde{x}=\left(x_{1}, \ldots, x_{n-1}\right)$ so that $x=\left(\tilde{x}, x_{n}\right)$. For $H \in \mathbb{R}$ let $U_{H}:=\left\{x: x_{n}>H\right\}$ and $\Gamma_{H}:=\left\{x: x_{n}=H\right\}$. Let $D \subset \mathbb{R}^{n}$ be a connected open set such that for some constants $f_{-}<f_{+}$it holds that

$$
U_{f_{+}} \subset D \subset U_{f_{-}} .
$$

This definition of $D$ (the domain of the acoustic field) allows the rough surface $\Gamma=\partial D$ to be more general than the graph of a function. The variational problem will be posed on the open set $S_{H}:=D \backslash \bar{U}_{H}$, for some $H \geq f_{+}$, and we denote the unit outward normal to $S_{H}$ by $\nu$.

Given a source $g \in L^{2}(D)$ of compact support, the problem we wish to analyze is to find an acoustic field $u$ such that

$$
\Delta u+k^{2} u=g \quad \text { in } D,
$$




$$
u=0 \quad \text { on } \Gamma,
$$

and such that $u$ satisfies an appropriate radiation condition.

This problem has been studied in a rigorous manner by integral equation methods $[10,9,30,3,4,28,8]$ in the case when $\Gamma$ is the graph of a sufficiently smooth-bounded function $f$ so that

$$
\Gamma=\left\{\left(\tilde{x}, x_{n}\right): x_{n}=f(\tilde{x}), \quad \tilde{x} \in \mathbb{R}^{n-1}\right\}
$$

with $f$ at least bounded and continuous. The most general results are restricted to the $2 \mathrm{D}$ case $[10,9,30,3,4,28]$. In the case $n=2$ with $(2.2)$ understood in a distributional sense, a solution $u \in C^{1}(D) \cap C(\bar{D})$ is sought such that $u$ is bounded in every strip $S_{H}, H>f_{+}$, and such that $u$ satisfies the upward propagating radiation condition (UPRC) proposed in [10], which states that

$$
u(x)=2 \int_{\Gamma_{H}} \frac{\partial \Phi(x, y)}{\partial y_{n}} u(y) d s(y), \quad x \in U_{H},
$$

for all $H$ such that the support of $g$ is contained in $S_{H}$. Here the fundamental solution of the Helmholtz equation $\Phi$ is given by

$$
\Phi(x, y)= \begin{cases}\frac{\mathrm{i}}{4} H_{0}^{(1)}(k|x-y|), & n=2, \\ \frac{\exp (\mathrm{i} k|x-y|)}{4 \pi|x-y|}, & n=3,\end{cases}
$$

for $x, y \in \mathbb{R}^{n}, x \neq y$, where $H_{0}^{(1)}$ is the Hankel function of the first kind of order zero. Under the assumption that $\Gamma$ is Lipschitz (i.e., that $f \in C^{0,1}\left(\mathbb{R}^{n-1}\right)$ ), and that $\Gamma$ is piecewise Lyapunov, uniqueness of solution is shown in the $2 \mathrm{D}$ case in [11].

To show existence of solution to (2.2)-(2.5) one approach is to first convert the boundary value problem to an equivalent Dirichlet boundary value problem. To do this we need to split $u$ into an incident and scattered field. Introducing the Dirichlet Green's function for the half-space $U_{a}$, defined by

$$
\mathcal{G}_{a}(x, y)=\Phi(x, y)-\Phi\left(x, y_{a}^{\prime}\right),
$$

where $y_{a}^{\prime}$ is the reflection of $y$ in $\Gamma_{a}$, we define the incident field $u_{a}^{i}$, for $a<f_{-}$, by

$$
u_{a}^{i}(x)=-\int_{D} \mathcal{G}_{a}(x, y) g(y) d y .
$$

Then $u_{a}^{i} \in H_{\mathrm{loc}}^{2}\left(U_{a}\right)$ and satisfies (2.2) in a distributional sense in $U_{a}$. Choosing $a<f_{-}$, we write $u$ as

$$
u=u_{a}^{i}+u^{s}
$$

and seek the scattered field $u^{s} \in C^{2}(D) \cap C(\bar{D})$ that satisfies

$$
\begin{array}{rlrl}
\Delta u^{s}+k^{2} u^{s} & =0 & & \text { in } D, \\
u^{s}=G & & \text { on } \Gamma,
\end{array}
$$

where $G:=-\left.u_{a}^{i}\right|_{\Gamma}$. Then $u$, given by (2.6), satisfies (2.2)-(2.5) provided $u^{s}$ satisfies $(2.5)$. 
In the case $n=2$ and $f \in C^{1,1}(\mathbb{R})$ it has been shown, for arbitrary bounded and continuous data $G$, that this Dirichlet problem for $u^{s}$ has exactly one solution that satisfies the radiation condition (2.5) [10]. Moreover, in the case that $G=-\left.u_{a}^{i}\right|_{\Gamma}$ it holds that $G(x)=O\left(|x|^{-3 / 2}\right)$ as $|x| \rightarrow \infty$, and it is shown in $[9,3,4]$ that $u^{s}$ and $u$ inherit this property; precisely that $u(x)=O\left(|x|^{-3 / 2}\right)$ as $\left|x_{1}\right| \rightarrow \infty$ with $x_{2}=O(1)$. Thus $G \in L^{2}(\Gamma)$ and $u \in L^{2}\left(S_{H}\right)$ for each $H>f_{-}$. It follows from local regularity estimates up to the boundary that $u \in C^{1}(\bar{D})$. Further, by an application of Green's theorem, the Helmholtz equation, and the a priori estimates up to the boundary of [11, Theorem 3.1], it follows also that $u \in H^{1}\left(S_{H}\right)$ for every $H>f_{+}$. This in turn implies that $\left.u\right|_{\Gamma_{H}} \in H^{1 / 2}\left(\Gamma_{H}\right) \subset L^{2}\left(\Gamma_{H}\right)$ for every $H \geq f_{+}$.

In the case that $\left.u\right|_{\Gamma_{H}} \in L^{2}\left(\Gamma_{H}\right)$ we can rewrite (2.5) in terms of the Fourier transform of $\left.u\right|_{\Gamma_{H}}$. For $\phi \in L^{2}\left(\Gamma_{H}\right)$, which we identify with $L^{2}\left(\mathbb{R}^{n-1}\right)$, we denote by $\hat{\phi}=\mathcal{F} \phi$ the Fourier transform of $\phi$ which we define by

$$
\mathcal{F} \phi(\xi)=(2 \pi)^{-(n-1) / 2} \int_{\mathbb{R}^{n-1}} \exp (-\mathrm{i} \tilde{x} \cdot \xi) \phi(\tilde{x}) d \tilde{x}, \quad \xi \in \mathbb{R}^{n-1} .
$$

Our choice of normalization of the Fourier transform ensures that $\mathcal{F}$ is a unitary operator on $L^{2}\left(\mathbb{R}^{n-1}\right)$ so that, for $\phi, \psi \in L^{2}\left(\mathbb{R}^{n-1}\right)$,

$$
\int_{\mathbb{R}^{n-1}} \phi \bar{\psi} d \tilde{x}=\int_{\mathbb{R}^{n-1}} \hat{\phi} \overline{\hat{\psi}} d \xi
$$

If $F_{H}:=\left.u\right|_{\Gamma_{H}} \in L^{2}\left(\Gamma_{H}\right)$, then (see [11, 5] in the case $\left.n=2\right)(2.5)$ can be rewritten as

$$
u(x)=\frac{1}{(2 \pi)^{(n-1) / 2}} \int_{\mathbb{R}^{n-1}} \exp \left(\mathrm{i}\left[\left(x_{n}-H\right) \sqrt{k^{2}-\xi^{2}}+\tilde{x} \cdot \xi\right]\right) \hat{F}_{H}(\xi) d \xi, \quad x \in U_{H}
$$

In this equation $\sqrt{k^{2}-\xi^{2}}=\mathrm{i} \sqrt{\xi^{2}-k^{2}}$, when $|\xi|>k$.

Equation (2.9) is a representation for $u$, in the upper half-plane $U_{H}$, as a superposition of upward propagating homogeneous and inhomogeneous plane waves. A requirement that (2.9) holds is commonly used (e.g., [13]) as a formal radiation condition in the physics and engineering literature on rough surface scattering. The meaning of $(2.9)$ is clear when $F_{H} \in L^{2}\left(\mathbb{R}^{n-1}\right)$ so that $\hat{F}_{H} \in L^{2}\left(\mathbb{R}^{n-1}\right)$; indeed the integral (2.9) exists in the Lebesgue sense for all $x \in U_{H}$. Recently Arens and Hohage [5] have explained, in the case $n=2$, in what precise sense (2.9) can be understood when $F_{H} \in B C\left(\Gamma_{H}\right)$, the space of bounded continuous functions on $\Gamma_{H}$, so that $\hat{F}_{H}$ must be interpreted as a tempered distribution.

The above discussion motivates the following precise formulation of problem (2.2)(2.3). Let $H_{0}^{1}(D)$ denote the standard Sobolev space, the completion of $C_{0}^{\infty}(D)$ in the norm $\|\cdot\|_{H^{1}(D)}$ defined by $\|u\|_{H^{1}(D)}=\left\{\int_{D}\left(|\nabla u|^{2}+|u|^{2}\right) d x\right\}^{1 / 2}$. The main function space in which we set our problem will be the Hilbert space $V_{H}$, defined, for $H \geq f_{+}$, by

$$
V_{H}:=\left\{\left.\phi\right|_{S_{H}}: \phi \in H_{0}^{1}(D)\right\},
$$

on which we impose the wave number dependent scalar product $(u, v)_{V_{H}}:=\int_{S_{H}}(\nabla u$. $\left.\overline{\nabla v}+k^{2} u \bar{v}\right) d x$ and norm $\|u\|_{V_{H}}=\left\{\int_{S_{H}}\left(|\nabla u|^{2}+k^{2}|u|^{2}\right) d x\right\}^{1 / 2}$.

The boundary value problem. Given $g \in L^{2}(D)$, whose support lies in $\overline{S_{H}}$, for some $H \geq f_{+}$, find $u: D \rightarrow \mathbb{C}$ such that $\left.u\right|_{S_{a}} \in V_{a}$ for every $a>f_{+}$,

$$
\Delta u+k^{2} u=g \quad \text { in } D
$$


in a distributional sense, and the radiation condition (2.9) holds with $F_{H}=\left.u\right|_{\Gamma_{H}}$.

REMARK 2.1. We note that, as one would hope, the solutions of the above problem do not depend on the choice of $H$. Precisely, if $u$ is a solution to the above problem for one value of $H \geq f_{+}$for which $\operatorname{supp} g \subset \overline{S_{H}}$, then $u$ is a solution for all $H \geq f_{+}$ with this property. To see that this is true is a matter of showing that if (2.9) holds for one $H$ with $\operatorname{supp} g \subset \overline{S_{H}}$, then (2.9) holds for all $H$ with this property. It is shown in Lemma 2.2 that if (2.9) holds, with $F_{H}=\left.u\right|_{\Gamma_{H}}$, for some $H \geq f_{+}$, then it holds for all larger values of $H$. One way to show that (2.9) holds also for every smaller value of $H, \tilde{H}$ say, for which $\tilde{H} \geq f_{+}$and $\operatorname{supp} g \subset \overline{S_{\tilde{H}}}$, is to consider the function

$v(x):=u(x)-\frac{1}{(2 \pi)^{(n-1) / 2}} \int_{\mathbb{R}^{n-1}} \exp \left(\mathrm{i}\left[\left(x_{n}-\tilde{H}\right) \sqrt{k^{2}-\xi^{2}}+\tilde{x} \cdot \xi\right]\right) \hat{F}_{\tilde{H}}(\xi) d \xi, \quad x \in U_{\tilde{H}}$,

with $F_{\tilde{H}}:=\left.u\right|_{\Gamma_{\tilde{H}}}$, and show that $v$ is identically zero. To see this we note that, by Lemma 2.2, $v$ satisfies the above boundary value problem with $D=U_{\tilde{H}}$ and $g=0$. That $v \equiv 0$, then follows from Theorem 4.1 .

As indicated in the above discussion, it is known that the above boundary value problem has a solution in the case $n=2$ when $\Gamma$ is the graph of a sufficiently smooth function. A main result of this paper is to prove that the boundary value problem is uniquely solvable, both in two and three dimensions, under much more general conditions on the boundary $\Gamma$. Moreover, we provide explicit estimates of the norm of the solution in the strip $S_{H}$ as a function of the dimensionless wave number

$$
\kappa=k\left(H-f_{-}\right) .
$$

We now derive a variational formulation of the boundary value problem above. To derive this alternative formulation we require a preliminary lemma. In this lemma and subsequently we use standard fractional Sobolev space notation, except that we adopt a wave number dependent norm, equivalent to the usual norm, and reducing to the usual norm if the unit of length measurement is chosen so that $k=1$. Thus, identifying $\Gamma_{H}$ with $\mathbb{R}^{n-1}, H^{s}\left(\Gamma_{H}\right)$, for $s \in \mathbb{R}$, denotes the completion of $C_{0}^{\infty}\left(\Gamma_{H}\right)$ in the norm $\|\cdot\|_{H^{s}\left(\Gamma_{H}\right)}$ defined by

$$
\|\phi\|_{H^{s}\left(\Gamma_{H}\right)}=\left(\int_{\mathbb{R}^{n-1}}\left(k^{2}+\xi^{2}\right)^{s}|\mathcal{F} \phi(\xi)|^{2} d \xi\right)^{1 / 2} .
$$

We recall [1] that, for all $a>H \geq f_{+}$, there exist continuous embeddings $\gamma_{+}$: $H^{1}\left(U_{H} \backslash U_{a}\right) \rightarrow H^{1 / 2}\left(\Gamma_{H}\right)$ and $\gamma_{-}: V_{H} \rightarrow H^{1 / 2}\left(\Gamma_{H}\right)$ (the trace operators) such that $\gamma_{ \pm} \phi$ coincides with the restriction of $\phi$ to $\Gamma_{H}$ when $\phi$ is $C^{\infty}$. In the case when $H=f_{+}$, when $\Gamma_{H}$ may not be a subset of the boundary of $S_{H}$ (if part of $\partial D$ coincides with $\left.\Gamma_{H}\right)$ we understand this trace by first extending $\phi \in V_{H}$ by zero to $U_{f_{-}} \backslash \bar{U}_{f_{+}}$. We recall also that if $u_{+} \in H^{1}\left(U_{H} \backslash U_{a}\right), u_{-} \in V_{H}$, and $\gamma_{+} u_{+}=\gamma_{-} u_{-}$, then $v \in V_{a}$, where $v(x):=u_{+}(x), x \in U_{H} \backslash U_{a},:=u_{-}(x), x \in S_{H}$. Conversely, if $v \in V_{a}$ and $u_{+}:=\left.v\right|_{U_{H} \backslash U_{a}}, u_{-}:=\left.v\right|_{S_{H}}$, then $\gamma_{+} v_{+}=\gamma_{-} v_{-}$. We introduce the operator $T$, which will prove to be a Dirichlet to Neumann map on $\Gamma_{H}$ (see (2.20)) defined by

$$
T:=\mathcal{F}^{-1} M_{z} \mathcal{F}
$$

where $M_{z}$ is the operation of multiplying by

$$
z(\xi):= \begin{cases}-\mathrm{i} \sqrt{k^{2}-\xi^{2}} & \text { if }|\xi| \leq k, \\ \sqrt{\xi^{2}-k^{2}} & \text { for }|\xi|>k\end{cases}
$$


We shall prove shortly in Lemma 2.4 that $T: H^{1 / 2}\left(\Gamma_{H}\right) \rightarrow H^{-1 / 2}\left(\Gamma_{H}\right)$ and is bounded.

Lemma 2.2. If (2.9) holds, with $F_{H} \in H^{1 / 2}\left(\Gamma_{H}\right)$, then $u \in H^{1}\left(U_{H} \backslash U_{a}\right) \cap C^{2}\left(U_{H}\right)$, for every $a>H$,

$$
\Delta u+k^{2} u=0 \quad \text { in } U_{H}
$$

$\gamma_{+} u=F_{H}$, and

$$
\int_{\Gamma_{H}} \bar{v} T \gamma_{+} u d s+k^{2} \int_{U_{H}} u \bar{v} d x-\int_{U_{H}} \nabla u \cdot \nabla \bar{v} d x=0, \quad v \in C_{0}^{\infty}(D) .
$$

Further, the restrictions of $u$ and $\nabla u$ to $\Gamma_{a}$ are in $L^{2}\left(\Gamma_{a}\right)$ for all $a>H$, and

$$
\int_{\Gamma_{a}}\left[\left|\frac{\partial u}{\partial x_{n}}\right|^{2}-\left|\nabla_{\tilde{x}} u\right|^{2}+k^{2}|u|^{2}\right] d s \leq 2 k \Im \int_{\Gamma_{a}} \bar{u} \frac{\partial u}{\partial x_{n}} d s .
$$

Moreover, for all $a>H$, where $F_{a} \in H^{1 / 2}\left(\Gamma_{a}\right)$ denotes the restriction of $u$ to $\Gamma_{a}$, (2.9) holds with $H$ replaced by $a$.

Proof. If $F_{H} \in L^{2}\left(\Gamma_{H}\right)$, then, as a function of $\xi, \exp \left(i\left[\left(x_{n}-H\right) \sqrt{k^{2}-\xi^{2}}+\tilde{x}\right.\right.$. $\xi]) \hat{F}_{H}(\xi)\left(1+\xi^{2}\right)^{s} \in L^{1}\left(\mathbb{R}^{n-1}\right)$ for every $x \in U_{H}$ and $s \geq 0$. It follows that (2.9) is well-defined for every $x \in U_{H}$, and that $u \in C^{2}\left(U_{H}\right)$, with all partial derivatives computed by differentiating under the integral sign, so that $\Delta u+k^{2} u=0$ in $U_{H}$. Thus, for $a>H$ and almost all $\xi \in \mathbb{R}^{n-1}$,

$$
\begin{aligned}
\mathcal{F}\left(\left.u\right|_{\Gamma_{a}}\right)(\xi) & =\exp \left(\mathrm{i}(a-H) \sqrt{k^{2}-\xi^{2}}\right) \hat{F}_{H}(\xi), \\
\mathcal{F}\left(\left.\frac{\partial u}{\partial x_{n}}\right|_{\Gamma_{a}}\right)(\xi) & =\mathrm{i} \sqrt{k^{2}-\xi^{2}} \exp \left(\mathrm{i}(a-H) \sqrt{k^{2}-\xi^{2}}\right) \hat{F}_{H}(\xi), \\
\mathcal{F}\left(\left.\nabla_{\tilde{x}} u\right|_{\Gamma_{a}}\right)(\xi) & =\mathrm{i} \xi \exp \left(\mathrm{i}(a-H) \sqrt{k^{2}-\xi^{2}}\right) \hat{F}_{H}(\xi) .
\end{aligned}
$$

Therefore, by the Plancherel identity (2.8), $\left.u\right|_{\Gamma_{a}},\left.\nabla u\right|_{\Gamma_{a}} \in L^{2}\left(\Gamma_{a}\right)$ with

$$
\int_{\Gamma_{a}}|u|^{2} d s=\int_{\mathbb{R}^{n-1}}\left|\exp \left(2 \mathrm{i}(a-H) \sqrt{k^{2}-\xi^{2}}\right)\right|\left|\hat{F}_{H}(\xi)\right|^{2} d \xi \leq \int_{\Gamma_{H}}\left|F_{H}\right|^{2} d s
$$

and

$$
\int_{\Gamma_{a}}|\nabla u|^{2} d s \leq \int_{\mathbb{R}^{n-1}}\left[\left|k^{2}-\xi^{2}\right|+\xi^{2}\right]\left|\exp \left(2 \mathrm{i}(a-H) \sqrt{k^{2}-\xi^{2}}\right)\right|\left|\hat{F}_{H}(\xi)\right|^{2} d \xi
$$

while

$$
\int_{\Gamma_{a}}\left[\left|\frac{\partial u}{\partial x_{n}}\right|^{2}-\left|\nabla_{\tilde{x}} u\right|^{2}+k^{2}|u|^{2}\right] d s=2 \int_{|\xi|<k}\left(k^{2}-\xi^{2}\right)\left|\hat{F}_{H}(\xi)\right|^{2} d \xi
$$

and

$$
\Im \int_{\Gamma_{a}} \bar{u} \frac{\partial u}{\partial x_{n}} d s=\int_{|\xi|<k} \sqrt{k^{2}-\xi^{2}}\left|\hat{F}_{H}(\xi)\right|^{2} d \xi .
$$

Thus (2.14) holds and

$$
\int_{U_{H} \backslash U_{a}}|u|^{2} d x \leq(a-H) \int_{\Gamma_{H}}\left|F_{H}\right|^{2} d s .
$$


Further, from (2.17) it follows that

$$
\begin{aligned}
\int_{U_{H} \backslash U_{a}}|\nabla u|^{2} d x \leq & (a-H) k^{2} \int_{|\xi|<k}\left|\hat{F}_{H}(\xi)\right|^{2} d \xi \\
& +\int_{|\xi|>k} \xi^{2} \frac{1-\exp \left(-2[a-H] \sqrt{\xi^{2}-k^{2}}\right)}{\sqrt{\xi^{2}-k^{2}}}\left|\hat{F}_{H}(\xi)\right|^{2} d \xi \\
\leq & \int_{\mathbb{R}^{n-1}}\left(4(a-H) k^{2}+\sqrt{2}|\xi|\right)\left|\hat{F}_{H}(\xi)\right|^{2} d \xi
\end{aligned}
$$

since $1-\mathrm{e}^{-z} \leq z$ for $z \geq 0$ and $\sqrt{\xi^{2}-k^{2}} \geq|\xi| / \sqrt{2}$ for $\xi^{2} \geq 2 k^{2}$. Thus $u \in H^{1}\left(U_{H} \backslash U_{a}\right)$ if $F_{H} \in H^{1 / 2}\left(\Gamma_{H}\right)$. That $\left.u\right|_{\Gamma_{H}}=F_{H}$ is clear when $F_{H} \in C_{0}^{\infty}\left(\Gamma_{H}\right)$, and $\gamma_{+} u=F_{H}$ for all $F_{H} \in H^{1 / 2}\left(\Gamma_{H}\right)$ follows from the continuity of $\gamma_{+},(2.18)$ and (2.19), and the density of $C_{0}^{\infty}\left(\Gamma_{H}\right)$ in $H^{1 / 2}\left(\Gamma_{H}\right)$. Similarly, in the case that $F_{H} \in C_{0}^{\infty}\left(\Gamma_{H}\right)$ so that $u \in C^{\infty}\left(\overline{U_{H}}\right)$, it is easily seen that

$$
T \gamma_{+} u=-\partial u /\left.\partial x_{n}\right|_{\Gamma_{H}},
$$

and (2.13) follows by Green's theorem. The same equation for the general case follows from the density of $C_{0}^{\infty}\left(\Gamma_{H}\right)$ in $H^{1 / 2}\left(\Gamma_{H}\right),(2.18)$ and (2.19), and the continuity of the operator $T$.

That (2.9) holds with $H$ replaced by $a$, for all $a>H$, is clear from (2.15).

Now suppose that $u$ satisfies the boundary value problem. Then $\left.u\right|_{S_{a}} \in V_{a}$ for every $a>f_{+}$and, by definition, since $\Delta u+k^{2} u=g$ in a distributional sense,

$$
\int_{D}\left[g \bar{v}+\nabla u \cdot \nabla \bar{v}-k^{2} u \bar{v}\right] d x=0, \quad v \in C_{0}^{\infty}(D) .
$$

Applying Lemma 2.2, and defining $w:=\left.u\right|_{S_{H}}$, it follows that

$$
\int_{S_{H}}\left[g \bar{v}+\nabla w \cdot \nabla \bar{v}-k^{2} w \bar{v}\right] d x+\int_{\Gamma_{H}} \bar{v} T \gamma_{-} w d s=0, \quad v \in C_{0}^{\infty}(D) .
$$

From the denseness of $\left\{\left.\phi\right|_{S_{H}}: \phi \in C_{0}^{\infty}(D)\right\}$ in $V_{H}$ and the continuity of $\gamma_{-}$and $T$, it follows that this equation holds for all $v \in V_{H}$.

Let $\|\cdot\|_{2}$ and $(\cdot, \cdot)$ denote the norm and scalar product on $L^{2}\left(S_{H}\right)$ so that $\|v\|_{2}=$ $\sqrt{\int_{S_{H}}|v|^{2} d x}$ and

$$
(u, v)=\int_{S_{H}} u \bar{v} d x
$$

and define the sesquilinear form $b: V_{H} \times V_{H} \rightarrow \mathbb{C}$ by

$$
b(u, v)=(\nabla u, \nabla v)-k^{2}(u, v)+\int_{\Gamma_{H}} \gamma_{-} \bar{v} T \gamma_{-} u d s .
$$

Then we have shown that if $u$ satisfies the boundary value problem, then $w:=\left.u\right|_{S_{H}}$ is a solution of the following variational problem: find $u \in V_{H}$ such that

$$
b(u, v)=-(g, v), \quad v \in V_{H} .
$$

Conversely, suppose that $w$ is a solution to the variational problem and define $u(x)$ to be $w(x)$ in $S_{H}$ and to be the right-hand side of (2.9), with $F_{H}:=\gamma_{-} w$, in 
$U_{H}$. Then, by Lemma $2.2, u \in H^{1}\left(U_{H} \backslash U_{a}\right)$ for every $a>H$ with $\gamma_{+} u=F_{H}=\gamma_{-} w$. Thus $\left.u\right|_{S_{a}} \in V_{a}, a \geq f_{+}$. Further, from (2.13) and (2.23) it follows that (2.21) holds so that $\Delta u+k^{2} u=g$ in $D$ in a distributional sense. Thus $u$ satisfies the boundary value problem.

We have thus proved the following theorem.

THEOREM 2.3. If $u$ is a solution of the boundary value problem, then $\left.u\right|_{S_{H}}$ satisfies the variational problem, Conversely, if u satisfies the variational problem, $F_{H}:=\gamma_{-} u$, and the definition of $u$ is extended to $D$ by setting $u(x)$ equal to the right-hand side of (2.9), for $x \in U_{H}$, then the extended function satisfies the boundary value problem, with $g$ extended by zero from $S_{H}$ to $D$.

It remains to prove the mapping properties of $T$.

Lemma 2.4. The Dirichlet-to-Neumann map $T$ defined by (2.12) is a bounded linear map from $H^{1 / 2}\left(\Gamma_{H}\right)$ to $H^{-1 / 2}\left(\Gamma_{H}\right)$ with $\|T\|=1$.

Proof. From the definitions of $T$ and the Sobolev norms we see that, as a map from $H^{1 / 2}\left(\Gamma_{H}\right)$ to $H^{-1 / 2}\left(\Gamma_{H}\right)$,

$$
\|T\|=\max _{\xi \in \mathbb{R}^{n-1}} \frac{\left|\sqrt{k^{2}-\xi^{2}}\right|}{\left|\sqrt{k^{2}+\xi^{2}}\right|}=1 .
$$

While the variational formulation (2.23) does not appear to have been studied previously, the analogous weak formulation for the $2 \mathrm{D}$ diffraction grating case has recently been studied in [16], as mentioned in the introduction. The diffraction grating case, with $f$ periodic and $g$ quasi-periodic with the same period, is significantly simpler because the variational problem can be formulated on a bounded domain (one period of the strip $S_{H}$ ) and the corresponding sesquilinear form on this bounded domain satisfies a Gårding inequality. Standard methods of analysis thus apply, in particular, existence follows from uniqueness via the Fredholm alternative. But we note that, even in the diffraction grating case, establishing uniqueness for arbitrary Lipschitz domains $D$ ( $f$ Lipschitz) requires careful and ingenious arguments [16] which are not required for scattering by bounded domains. Indeed, uniqueness does not hold in all cases in which $\partial D$ is not the graph of a function, as is shown by the example in Gotlib [18].

3. Analysis of the variational problem for low frequency. In this section we shall derive preliminary results and bounds used throughout, and will analyze (2.23) when $k$ is sufficiently small that $b$ is $V_{H}$-elliptic (we shall give an explicit bound for $k$ to guarantee this). An attraction of our results for low wave number, in contrast to our results in section 4 for larger wave number, is that we require no additional assumption on the domain, except that $\kappa$, given by (2.11), be sufficiently small. We note also that the bounds we establish for $\kappa$ small in Theorem 3.1 are somewhat sharper than those which can be established as valid for general $\kappa$ by the techniques of the next section. From the point of view of numerical solution by, e.g., finite element methods, the ellipticity we establish for small $k$ is of course highly desirable, guaranteeing, by Céa's lemma, unique existence and stability of the numerical solution method.

Let $V_{H}^{*}$ denote the dual space of $V_{H}$, i.e., the space of continuous antilinear functionals on $V_{H}$. Then our analysis will also apply to the following slightly more general problem: given $\mathcal{G} \in V_{H}^{*}$ find $u \in V_{H}$ such that

$$
b(u, v)=\mathcal{G}(v), \quad v \in V_{H} .
$$

We shall prove the following theorem. 
THEOREM 3.1. Suppose the wave number $k$ satisfies $k<\sqrt{2} /\left(H-f_{-}\right)$(equivalently $\kappa<\sqrt{2}$ ). Then the sesquilinear form $b$ is $V_{H}$-elliptic so that the variational problem (3.1) is uniquely solvable, and the solution satisfies the estimate

$$
\|u\|_{V_{H}} \leq C\|\mathcal{G}\|_{V_{H}^{*}}
$$

where the constant $C$ satisfies

$$
C \leq \frac{1+\kappa^{2} / 2}{1-\kappa^{2} / 2}
$$

In particular, the scattering problem (2.23) is uniquely solvable and the solution satisfies the bound

$$
k\|u\|_{V_{H}} \leq \frac{\kappa}{\sqrt{2}} \frac{1+\kappa^{2} / 2}{1-\kappa^{2} / 2}\|g\|_{2} .
$$

In order to prove Theorem 3.1 we establish a sequence of lemmas which are of some independent interest and are used extensively in the rest of the paper. The first two concern the Dirichlet to Neumann map $T$ and the trace operator $\gamma_{-}$and will be proved using the Fourier transform (2.7).

Lemma 3.2. For all $\phi, \psi \in H^{1 / 2}\left(\Gamma_{H}\right)$,

$$
\int_{\Gamma_{H}} \phi T \psi d s=\int_{\Gamma_{H}} \psi T \phi d s
$$

For all $\phi \in H^{1 / 2}\left(\Gamma_{H}\right)$,

$$
\Re \int_{\Gamma_{H}} \bar{\phi} T \phi d s \geq 0, \quad \Im \int_{\Gamma_{H}} \bar{\phi} T \phi d s \leq 0 .
$$

Proof. Let $\hat{\phi}=\mathcal{F} \phi, \hat{\psi}=\mathcal{F} \psi$. Then $\mathcal{F}(T \phi)=z \hat{\phi}$. Thus, using the Plancherel identity (2.8) and since $\hat{\bar{\psi}}(\xi)=\overline{\hat{\psi}(-\xi)}$ and $z$ is even,

$$
\int_{\Gamma_{H}} \psi T \phi d s=\int_{\mathbb{R}^{n-1}} \hat{\psi}(-\xi) z(\xi) \hat{\phi}(\xi) d \xi=\int_{\mathbb{R}^{n-1}} \hat{\psi}(\xi) z(\xi) \hat{\phi}(-\xi) d \xi=\int_{\Gamma_{H}} \phi T \psi d s .
$$

In particular, putting $\psi=\bar{\phi}$,

$$
\begin{aligned}
\int_{\Gamma_{H}} \bar{\phi} T \phi d s & =\int_{\mathbb{R}^{n-1}} z(\xi)|\hat{\phi}(\xi)|^{2} d \xi \\
& =\int_{|\xi|>k} \sqrt{\xi^{2}-k^{2}}|\hat{\phi}(\xi)|^{2} d \xi-\mathrm{i} \int_{|\xi|<k} \sqrt{k^{2}-\xi^{2}}|\hat{\phi}(\xi)|^{2} d \xi,
\end{aligned}
$$

from which the second result follows.

The above lemma implies that $b(\cdot, \cdot)$ has the following important symmetry property.

Corollary 3.3. For all $u, v \in V_{H}$,

$$
b(v, u)=b(\bar{u}, \bar{v}) .
$$


Lemma 3.4. For all $u \in V_{H}$,

$$
\left\|\gamma_{-} u\right\|_{H^{1 / 2}\left(\Gamma_{H}\right)} \leq\|u\|_{V_{H}}
$$

and

$$
\|u\|_{2} \leq \frac{H-f_{-}}{\sqrt{2}}\left\|\frac{\partial u}{\partial x_{n}}\right\|_{2}
$$

Proof. For $u \in C_{0}^{\infty}(D) \subset C_{0}^{\infty}\left(U_{f_{-}}\right)$and defining $\hat{u}\left(\xi, x_{n}\right)=\left(\mathcal{F} u\left(\cdot, x_{n}\right)\right)(\xi)$, we have

$$
|\hat{u}(\xi, H)|^{2}=\int_{f_{-}}^{H} \frac{\partial}{\partial x_{n}}\left|\hat{u}\left(\xi, x_{n}\right)\right|^{2} d x_{n}=2 \Re \int_{f_{-}}^{H} \overline{\hat{u}\left(\xi, x_{n}\right)} \frac{\partial}{\partial x_{n}} \hat{u}\left(\xi, x_{n}\right) d x_{n} .
$$

Thus, if $S=\mathbb{R}^{n-1} \times\left(f_{-}, H\right)$,

$$
\begin{aligned}
\|u\|_{H^{1 / 2}\left(\Gamma_{H}\right)}^{2} & =\int_{\mathbb{R}^{n-1}}\left|\sqrt{\xi^{2}+k^{2}}\right||\hat{u}(\xi, H)|^{2} d \xi \\
& \leq 2 \int_{S}\left|\sqrt{\xi^{2}+k^{2}}\right| \hat{u}\left(\xi, x_{n}\right)|| \frac{\partial}{\partial x_{n}} \hat{u}\left(\xi, x_{n}\right) \mid d \xi d x_{n} \\
& \leq 2\left\{\int_{S}\left|\xi^{2}+k^{2}\right|\left|\hat{u}\left(\xi, x_{n}\right)\right|^{2} d \xi d x_{n}\right\}^{1 / 2}\left\{\int_{S}\left|\frac{\partial}{\partial x_{n}} \hat{u}\left(\xi, x_{n}\right)\right|^{2} d \xi d x_{n}\right\}^{1 / 2}
\end{aligned}
$$

Now, by Parseval's theorem,

$$
\begin{aligned}
\int_{S} \xi^{2}\left|\hat{u}\left(\xi, x_{n}\right)\right|^{2} d \xi d x_{n} & =\int_{S}\left|\mathcal{F}\left(\nabla_{\tilde{x}} u\left(\cdot, x_{n}\right)\right)(\xi)\right|^{2} d \xi d x_{n} \\
& =\int_{S}\left|\nabla_{\tilde{x}} u(x)\right|^{2} d x
\end{aligned}
$$

Applying Parseval's theorem again, and since $2 \sqrt{a b} \leq a+b$ for $a, b \geq 0$,

$$
\|u\|_{H^{1 / 2}\left(\Gamma_{H}\right)}^{2} \leq 2\left\{\int_{S}\left\{k^{2}|u(x)|^{2}+\left|\nabla_{\tilde{x}} u(x)\right|^{2}\right\} d x \int_{S}\left|\frac{\partial}{\partial x_{n}} u(x)\right|^{2} d x\right\}^{1 / 2} \leq\|u\|_{V_{H}}^{2} .
$$

Further, using the fact that $u \in C_{0}^{\infty}\left(U_{f_{-}}\right)$, for $x \in S$,

$$
|u(x)|^{2}=\left|\int_{f_{-}}^{x_{n}} \frac{\partial}{\partial x_{n}} u(x) d x_{n}\right|^{2} \leq\left(x_{n}-f_{-}\right) \int_{f_{-}}^{H}\left|\frac{\partial}{\partial x_{n}} u(x)\right|^{2} d x_{n}
$$

so that, since $\int_{f_{-}}^{H}\left(x_{n}-f_{-}\right) d x_{n}=\left(H-f_{-}\right)^{2} / 2$,

$$
\int_{S}|u(x)|^{2} d x \leq \frac{\left(H-f_{-}\right)^{2}}{2} \int_{S}\left|\frac{\partial}{\partial x_{n}} u(x)\right|^{2} d x .
$$

Since the set $\left\{\left.v\right|_{S_{H}}: v \in C_{0}^{\infty}(D)\right\}$ is dense in $V_{H}$ these bounds hold for all $u \in V_{H}$.

We are now in a position to prove that the sesquilinear form $b(.,$.$) is bounded,$ establishing an explicit value for the bound. 
Lemma 3.5. For all $u, v \in V_{H}$,

$$
|b(u, v)| \leq 2\|u\|_{V_{H}}\|v\|_{V_{H}}
$$

so that the sesquilinear form $b(.,$.$) is bounded.$

Proof. From the definition of the sesquilinear form $b(.,$.$) and the Cauchy-Schwarz$ inequality, we have

$$
|b(u, v)| \leq\|\nabla u\|_{2}\|\nabla v\|_{2}+k^{2}\|u\|_{2}\|v\|_{2}+\left\|\gamma_{-} u\right\|_{H^{1 / 2}\left(\Gamma_{H}\right)}\|T\|\left\|\gamma_{-} v\right\|_{H^{1 / 2}\left(\Gamma_{H}\right)} .
$$

Applying the Cauchy-Schwarz inequality and Lemmas 2.4 and 3.4 we obtain the desired estimate.

Our last lemma of this section shows that the sesquilinear form $b(.,$.$) is V_{H}$-elliptic provided the wave number $k$ is not too large.

Lemma 3.6. For all $u \in V_{H}$,

$$
\Re b(u, u) \geq \frac{1-\kappa^{2} / 2}{1+\kappa^{2} / 2}\|u\|_{V_{H}}^{2} .
$$

Proof. By Lemma 3.2,

$$
\Re b(u, u) \geq\|u\|_{V_{H}}^{2}-2 k^{2}\|u\|_{2}^{2} .
$$

The result follows from Lemma 3.4, implying that $\|u\|_{V_{H}}^{2} \geq k^{2}\left(2 / \kappa^{2}+1\right)\|u\|_{2}^{2}$.

Using Lemmas 3.5 and 3.6 we can now prove Theorem 3.1.

Proof. By Lemma 3.6 and under the assumption of the theorem that $k<\sqrt{2} /(H-$ $f_{-}$) we see that $b(.,$.$) is V_{H^{-}}$-elliptic. Lemma 3.5 shows that $b(.,$.$) is bounded and hence$ by the Lax-Milgram lemma the existence of a unique solution $u$ to (3.1) is assured. The estimate (3.2) also follows from the Lax-Milgram lemma. In the particular case that $\mathcal{G}(v):=-(g, v)$, for some $g \in L^{2}\left(S_{H}\right)$, we have further, by the Cauchy-Schwarz inequality and Lemma 3.4, that

$$
\|\mathcal{G}\|_{V_{H}^{*}}=\sup _{v \in V_{H}} \frac{|(v, g)|}{\|v\|_{V_{H}}} \leq \sup _{v \in V_{H}} \frac{\|v\|_{2}\|g\|_{2}}{\|v\|_{V_{H}}} \leq \frac{H-f_{-}}{\sqrt{2}}\|g\|_{2}
$$

and (3.3) follows.

4. Analysis of the variational problem at arbitrary frequency. The sesquilinear form $b(.,$.$) is not V_{H}$-elliptic if the wave number $k$ is large. In this section we shall establish, with no restriction on the wave number but some additional constraint on the domain, that the boundary value problem and the equivalent variational problem are uniquely solvable by using the generalized Lax-Milgram theory of Babuška. The domains $D$ for which we will establish this result are those which, in addition to our assumption throughout that $U_{f_{+}} \subset D \subset U_{f_{-}}$, satisfy the condition that

$$
x \in D \Rightarrow x+s e_{n} \in D \quad \text { for all } s>0,
$$

where $e_{n}$ denotes the unit vector in the direction $x_{n}$. Condition (4.1) is satisfied if $\Gamma$ is the graph of a continuous function, but certainly does not require that this be the case. Nor does (4.1) impose any regularity on $\partial D$. Our main result in this section is the following. 
THEOREM 4.1. If (4.1) holds, then the variational problem (3.1) has a unique solution $u \in V_{H}$ for every $\mathcal{G} \in V_{H}^{*}$ and

$$
\|u\|_{V_{H}} \leq C\|\mathcal{G}\|_{V_{H}^{*}}
$$

where

$$
C=1+\sqrt{2} \kappa(\kappa+1)^{2} .
$$

In particular, the boundary value problem and the equivalent variational problem (2.23) have exactly one solution, and the solution satisfies the bound

$$
k\|u\|_{V_{H}} \leq \frac{\kappa}{\sqrt{2}}(\kappa+1)^{2}\|g\|_{2} .
$$

To apply the generalized Lax-Milgram theorem (e.g., [19, Theorem 2.15]) we need to show that $b$ is bounded, which we have done in Lemma 3.5; to establish the inf-sup condition that

$$
\beta:=\inf _{0 \neq u \in V_{H}} \sup _{0 \neq v \in V_{H}} \frac{|b(u, v)|}{\|u\|_{V_{H}}\|v\|_{V_{H}}}>0
$$

and to establish a "transposed" inf-sup condition. It follows easily from Corollary 3.3 that this transposed inf-sup condition follows automatically if (4.3) holds.

Lemma 4.2. If (4.3) holds, then for all nonzero $v \in V_{H}$,

$$
\sup _{0 \neq u \in V_{H}} \frac{|b(u, v)|}{\|u\|_{V_{H}}}>0 .
$$

Proof. If (4.3) holds and $v \in V_{H}$ is nonzero, then

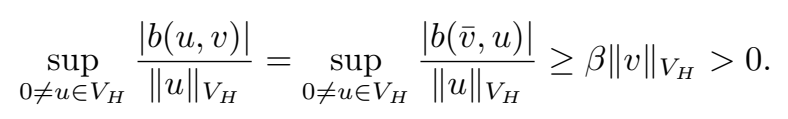

This proves the lemma.

The following result follows from [19, Theorem 2.15] and Lemmas 3.5 and 4.2.

COROLlaRY 4.3. If (4.3) holds, then the variational problem (3.1) has exactly one solution $u \in V_{H}$ for all $\mathcal{G} \in V_{H}^{*}$. Moreover,

$$
\|u\|_{V_{H}} \leq \beta^{-1}\|\mathcal{G}\|_{V_{H}^{*}}
$$

To show (4.3) we will establish an a priori bound for solutions of (3.1), from which the inf-sup condition will follow by the following easily established lemma (see [19, Remark 2.20]).

LEMma 4.4. Suppose that there exists $C>0$ such that for all $u \in V_{H}$ and $\mathcal{G} \in V_{H}^{*}$ satisfying (3.1) it holds that

$$
\|u\|_{V_{H}} \leq C\|\mathcal{G}\|_{V_{H}^{*}} .
$$

Then the inf-sup condition (4.3) holds with $\beta \geq C^{-1}$.

The following lemma reduces the problem of establishing (4.4) to that of establishing an a priori bound for solutions of the special case (2.23). 
Lemma 4.5. Suppose there exists $\tilde{C}>0$ such that for all $u \in V_{H}$ and $g \in L^{2}\left(S_{H}\right)$ satisfying (2.23) it holds that

$$
\|u\|_{V_{H}} \leq k^{-1} \tilde{C}\|g\|_{2} .
$$

Then, for all $u \in V_{H}$ and $\mathcal{G} \in V_{H}^{*}$ satisfying (3.1), the bound (4.4) holds with

$$
C \leq 1+2 \tilde{C}
$$

Proof. Suppose $u \in V_{H}$ is a solution of

$$
b(u, v)=\mathcal{G}(v), \quad v \in V_{H},
$$

where $\mathcal{G} \in V_{H}^{*}$. Let $b_{0}: V_{H} \times V_{H} \rightarrow \mathbb{C}$ be defined by

$$
b_{0}(u, v)=(\nabla u, \nabla v)+k^{2}(u, v)+\int_{\Gamma_{H}} \gamma_{-} \bar{v} T \gamma_{-} u d s, \quad u, v \in V_{H} .
$$

It follows from Lemma 3.2 that $b_{0}$ is $V_{H}$-elliptic, in fact that

$$
\Re b_{0}(v, v) \geq\|v\|_{V_{H}}^{2}, \quad v \in V_{H} .
$$

Thus the problem of finding $u_{0} \in V_{H}$ such that

$$
b_{0}\left(u_{0}, v\right)=\mathcal{G}(v), \quad v \in V_{H},
$$

has a unique solution which satisfies

$$
\left\|u_{0}\right\|_{V_{H}} \leq\|\mathcal{G}\|_{V_{H}^{*}}
$$

Furthermore, defining $w=u-u_{0}$ and using (4.6) and (4.7), we see that

$$
b(w, v)=b(u, v)-b\left(u_{0}, v\right)=\mathcal{G}(v)-\left(\mathcal{G}(v)-2 k^{2}\left(u_{0}, v\right)\right)=2 k^{2}\left(u_{0}, v\right)
$$

for all $v \in V_{H}$. Thus $w$ satisfies (2.23) with $g=-2 k^{2} u_{0}$. It follows, using (4.8), (4.5), and Lemma 3.4, that

$$
\|w\|_{V_{H}} \leq 2 k \tilde{C}\left\|u_{0}\right\|_{2} \leq 2 \tilde{C}\|\mathcal{G}\|_{V_{H}^{*}}
$$

The bound (4.4), with $C \leq 1+2 \tilde{C}$, follows from (4.8) and (4.9).

Following these preliminary lemmas we turn now to establishing the a priori bound (4.5), at first just for the case when $\Gamma$ is the graph of a smooth function. We recall that $\nu$ is the outward unit normal to $S_{H}$ and $\nu_{n}=\nu \cdot e_{n}$ is the $n$th (vertical) component of $\nu$.

Lemma 4.6. Suppose $\Gamma$ is given by (2.4) with $f \in C^{\infty}\left(\mathbb{R}^{n-1}\right)$. Let $H \geq f_{+}$, $g \in L^{2}\left(S_{H}\right)$ and suppose $w \in V_{H}$ satisfies

$$
b(w, \phi)=-(g, \phi) \quad \text { for all } \phi \in V_{H} .
$$

Then

$$
\|w\|_{V_{H}} \leq k^{-1} \tilde{C}\|g\|_{2}
$$


where $\tilde{C}=\frac{\kappa}{\sqrt{2}}(\kappa+1)^{2}$.

Proof. The proof of this lemma is motivated by [21, 12], where a Rellich identity is used to prove estimates for solutions of the Helmholtz equation posed on bounded domains, by the proofs of the basic inequalities for rough surface scattering problems in $[11,29]$, and by the estimates derived for the diffraction grating problem in [16].

Let $r=|\tilde{x}|$. For $A \geq 1$ let $\phi_{A} \in C_{0}^{\infty}(\mathbb{R})$ be such that $0 \leq \phi_{A} \leq 1, \phi_{A}(r)=1$ if $r \leq A$ and $\phi_{A}(r)=0$ if $r \geq A+1$ and finally such that $\left\|\phi_{A}^{\prime}\right\|_{\infty} \leq M$ for some fixed $M$ independent of $A$.

Extending the definition of $w$ to $D$ by defining $w$ in $U_{H}$ by (2.9) with $F_{H}:=\gamma_{-} w$, it follows from Theorem 2.3 that $w$ satisfies the boundary value problem with $g$ extended by zero from $S_{H}$ to $D$. By standard local regularity results [17] it holds, since $g \in L^{2}(D), w=0$ on $\Gamma$, and the boundary is smooth, that $w \in H_{\text {loc }}^{2}(D)$. Further, $w \in H^{2}\left(U_{b} \backslash U_{c}\right)$ for $c>b>f_{+}$(though $w \in H^{2}\left(S_{c}\right)$ is not clear without some further constraint on the behavior of $\Gamma$ at infinity). Moreover, by Lemma 2.2, $w$ is given by the right-hand side of (2.9) in $U_{b}$ for all $b>H$ if $H$ is replaced in (2.9) by $b$ and $F_{b}$ denotes the restriction of $w$ to $\Gamma_{b}$. Thus $w$ satisfies the boundary value problem with $H$ replaced by $b$ for all $b>H$, and so, by Theorem 2.3 ,

$$
\int_{S_{b}}\left(\nabla w \cdot \nabla \bar{v}-k^{2} w \bar{v}\right) d x=-\int_{\Gamma_{b}} \gamma_{-} \bar{v} T \gamma_{-} w d s-\int_{S_{b}} \bar{v} g d x
$$

for all $b \geq H$.

In view of this regularity and since $w$ satisfies the boundary value problem, we have, for all $a>H$,

$$
\begin{aligned}
& 2 \Re \int_{S_{a}} \phi_{A}(r)\left(x_{n}-f_{-}\right) g \frac{\partial \bar{w}}{\partial x_{n}} d x \\
&=2 \Re \int_{S_{a}} \phi_{A}(r)\left(x_{n}-f_{-}\right)\left(\Delta w+k^{2} w\right) \frac{\partial \bar{w}}{\partial x_{n}} d x \\
&=\int_{S_{a}}\left\{2 \Re\left\{\nabla \cdot\left(\phi_{A}(r)\left(x_{n}-f_{-}\right) \frac{\partial \bar{w}}{\partial x_{n}} \nabla w\right)\right\}-2 \phi_{A}(r)\left|\frac{\partial w}{\partial x_{n}}\right|^{2}\right. \\
&-\left(x_{n}-f_{-}\right) \phi_{A}(r) \frac{\partial|\nabla w|^{2}}{\partial x_{n}} \\
&\left.-2 \phi_{A}^{\prime}(r)\left(x_{n}-f_{-}\right) \frac{\tilde{x}}{|\tilde{x}|} \cdot \Re\left(\nabla_{\tilde{x}} w \frac{\partial \bar{w}}{\partial x_{n}}\right)+k^{2}\left(x_{n}-f_{-}\right) \phi_{A}(r) \frac{\partial|w|^{2}}{\partial x_{n}}\right\} d x .
\end{aligned}
$$

Using the divergence theorem and integration by parts,

$$
\begin{aligned}
2 \Re \int_{S_{a}} \phi_{A}(r)\left(x_{n}-f_{-}\right) g \frac{\partial \bar{w}}{\partial x_{n}} d x \\
=\left(a-f_{-}\right) \int_{\Gamma_{a}} \phi_{A}(r)\left\{\left|\frac{\partial w}{\partial x_{n}}\right|^{2}-\left|\nabla_{\tilde{x}} w\right|^{2}+k^{2}|w|^{2}\right\} d s \\
\quad-\int_{\Gamma}\left(x_{n}-f_{-}\right) \phi_{A}(r)\left\{\nu_{n}|\nabla w|^{2}-2 \Re\left(\frac{\partial \bar{w}}{\partial x_{n}} \frac{\partial w}{\partial \nu}\right)\right\} d s \\
\quad+\int_{S_{a}}\left\{\phi_{A}(r)\left(|\nabla w|^{2}-k^{2}|w|^{2}-2\left|\frac{\partial w}{\partial x_{n}}\right|^{2}\right)-2 \phi_{A}^{\prime}(r)\left(x_{n}-f_{-}\right) \Re\left(\frac{\partial \bar{w}}{\partial x_{n}} \frac{\partial w}{\partial r}\right)\right\} d x .
\end{aligned}
$$


Using the fact that $w=0$ on $\Gamma$ so that $\nabla w=(\partial w / \partial \nu) \nu$ and

$$
\frac{\partial w}{\partial x_{n}}=e_{n} \cdot \nabla w=e_{n} \cdot \nu \frac{\partial w}{\partial \nu}=\nu_{n} \frac{\partial w}{\partial \nu},
$$

and rearranging terms we find that

$$
\begin{aligned}
- & \int_{\Gamma} \phi_{A}(r)\left(x_{n}-f_{-}\right) \nu_{n}\left|\frac{\partial w}{\partial \nu}\right|^{2} d s+2 \int_{S_{a}} \phi_{A}(r)\left|\frac{\partial w}{\partial x_{n}}\right|^{2} d x \\
= & \left(a-f_{-}\right) \int_{\Gamma_{a}} \phi_{A}(r)\left\{\left|\frac{\partial w}{\partial x_{n}}\right|^{2}-\left|\nabla_{\tilde{x}} w\right|^{2}+k^{2}|w|^{2}\right\} d s \\
& +\int_{S_{a}}\left\{\phi_{A}(r)\left(|\nabla w|^{2}-k^{2}|w|^{2}\right)-2 \phi_{A}^{\prime}(r)\left(x_{n}-f_{-}\right) \Re\left(\frac{\partial \bar{w}}{\partial x_{n}} \frac{\partial w}{\partial r}\right)\right\} d x \\
& -2 \Re \int_{S_{a}} \phi_{A}(r)\left(x_{n}-f_{-}\right) g \frac{\partial \bar{w}}{\partial x_{n}} d x .
\end{aligned}
$$

We now wish to let $A \rightarrow \infty$. The only problem is the term involving $\phi_{A}^{\prime}$ which we estimate as follows. Let $S_{a}^{b}=\left\{x \in S_{a}:|\tilde{x}|<b\right\}$ for $b \geq 1$. Then

$$
\left|\int_{S_{a}}\left\{2 \phi_{A}^{\prime}(r)\left(x_{n}-f_{-}\right) \Re\left(\frac{\partial \bar{w}}{\partial x_{n}} \frac{\partial w}{\partial r}\right)\right\} d x\right| \leq 2 M\left(a-f_{-}\right) \int_{S_{a}^{A+1} \backslash \bar{S}_{a}^{A}}|\nabla w|^{2} d x \rightarrow 0
$$

as $A \rightarrow \infty$, where the convergence follows from the fact that $w \in H^{1}\left(S_{H}\right)$. In addition since $w \in H^{2}\left(U_{b} \backslash U_{c}\right)$, for $c>a>b>f_{+},\left.\nabla w\right|_{\Gamma_{H}} \in\left(H^{1 / 2}\left(\Gamma_{H}\right)\right)^{n}$. Thus, taking the limit as $A \rightarrow \infty$ in (4.12), and applying the Lebesgue dominated convergence and monotone convergence theorems, we see that

$$
\begin{aligned}
& -\int_{\Gamma}\left(x_{n}-f_{-}\right) \nu_{n}\left|\frac{\partial w}{\partial \nu}\right|^{2} d s+2 \int_{S_{a}}\left|\frac{\partial w}{\partial x_{n}}\right|^{2} d x \\
& =\left(a-f_{-}\right) \int_{\Gamma_{a}}\left\{\left|\frac{\partial w}{\partial x_{n}}\right|^{2}-\left|\nabla_{\tilde{x}} w\right|^{2}+k^{2}|w|^{2}\right\} d s \\
& \quad+\int_{S_{a}}\left(|\nabla w|^{2}-k^{2}|w|^{2}-2 \Re\left(\left(x_{n}-f_{-}\right) g \frac{\partial \bar{w}}{\partial x_{n}}\right)\right) d x .
\end{aligned}
$$

Now, since $w$ satisfies the boundary value problem, including the radiation condition (2.9), applying Lemma 2.2 it follows that

$$
\begin{aligned}
\int_{\Gamma_{a}}\left\{\left|\frac{\partial w}{\partial x_{n}}\right|^{2}-\left|\nabla_{\tilde{x}} w\right|^{2}+k^{2}|w|^{2}\right\} d s & \leq 2 k \Im \int_{\Gamma_{a}} \bar{w} \frac{\partial w}{\partial x_{n}} d s \\
& =-2 k \Im \int_{\Gamma_{a}} \gamma_{-} \bar{w} T \gamma_{-} w d s
\end{aligned}
$$

on applying the Plancherel identity (2.8), noting (2.15) and (2.16). Further, setting $v=w$ in (4.11), we get

$$
\int_{S_{b}}\left(|\nabla w|^{2}-k^{2}|w|^{2}\right) d x=-\int_{\Gamma_{b}} \gamma_{-} \bar{w} T \gamma_{-} w d s-\int_{S_{b}} g \bar{w} d x
$$


for $b \geq H$, so that, by Lemma 3.2 ,

$$
\int_{S_{b}}\left[|\nabla w|^{2}-k^{2}|w|^{2}\right] d x \leq-\Re \int_{S_{b}} g \bar{w} d x
$$

and

$$
-2 k \Im \int_{\Gamma_{b}} \gamma_{-} \bar{w} T \gamma_{-} w d s=2 k \Im \int_{S_{b}} g \bar{w} d x
$$

Using (4.17) in (4.14) and then using the resulting equation and (4.16) in (4.13) and noting that supp $g \subset \overline{S_{H}}$, we get that

$$
\begin{aligned}
-\int_{\Gamma}\left(x_{n}-f_{-}\right) \nu_{n}\left|\frac{\partial w}{\partial \nu}\right|^{2} d s+2 \int_{S_{H}}\left|\frac{\partial w}{\partial x_{n}}\right|^{2} d x \leq & 2\left(a-f_{-}\right) k \Im \int_{S_{H}} g \bar{w} d x \\
& -\Re \int_{S_{H}}\left[g \bar{w}+2\left(x_{n}-f_{-}\right) g \frac{\partial \bar{w}}{\partial x_{n}}\right] d x .
\end{aligned}
$$

Since this equation holds for all $a>H$ and $\nu_{n}<0$ on $\Gamma$, it follows by the CauchySchwarz inequality that

$$
2\left\|\frac{\partial w}{\partial x_{n}}\right\|_{2}^{2} \leq\left(2 \kappa\|w\|_{2}+\|w\|_{2}+2\left(H-f_{-}\right)\left\|\frac{\partial w}{\partial x_{n}}\right\|_{2}\right)\|g\|_{2} .
$$

Now using Lemma 3.4 to estimate $\|w\|_{2}$ we obtain

$$
\left\|\frac{\partial w}{\partial x_{n}}\right\|_{2} \leq\left(H-f_{-}\right)\left(\frac{1}{\sqrt{2}} \kappa+\frac{1}{2 \sqrt{2}}+1\right)\|g\|_{2}
$$

and use of Lemma 3.4 again shows that

$$
\|w\|_{2} \leq\left(H-f_{-}\right)^{2}\left(\frac{1}{2} \kappa+\frac{1}{4}+\frac{1}{\sqrt{2}}\right)\|g\|_{2} .
$$

Using the above inequality in (4.16) shows that

$$
\begin{aligned}
\|w\|_{V_{H}}^{2} & \leq 2 k^{2}\|w\|_{2}^{2}+\|g\|_{2}\|w\|_{2} \\
& \leq \frac{\left(H-f_{-}\right)^{2}}{4}\left(\frac{\kappa^{2}}{2}(2 \kappa+1+2 \sqrt{2})^{2}+2 \kappa+1+2 \sqrt{2}\right)\|g\|_{2}^{2} .
\end{aligned}
$$

Thus, for $\kappa \geq 1$,

$$
\|w\|_{V_{H}}^{2} \leq \frac{\left(H-f_{-}\right)^{2}}{2}(\kappa+1)^{4}\|g\|_{2}^{2} .
$$

The same bound holds for $\kappa<1$ by Theorem 3.1.

REMARK 4.7. The above argument works under milder assumptions on the boundary $\Gamma$, in particular that $\Gamma$ is the graph of a function $f \in C^{2}\left(\mathbb{R}^{n-1}\right)$, so that $\Gamma$ is of class $C^{2}$. This assumption is enough [17] to deduce the necessary local regularity result that $w \in H_{\text {loc }}^{2}(D)$.

Combining Lemmas 4.6, 4.5, and 4.4 with Corollary 4.3, we have the following result. 
LEMma 4.8. If $\Gamma$ satisfies the conditions of Lemma 4.6, then the variational problem (3.1) has a unique solution $u \in V_{H}$ for every $\mathcal{G} \in V_{H}^{*}$ and the solution satisfies the estimate (4.2).

REMARK 4.9. The above result, combined with Lemma 4.4, implies that $\beta$, the inf$\sup$ constant for $b(\cdot, \cdot)$, satisfies $\beta^{-1} \leq C=O\left(k^{3}\right)$ as $k \rightarrow \infty$. This high power of the wave number is, we suspect, not optimal. For an interior problem in a smooth starlike and bounded domain in $\mathbb{R}^{2}$ or $\mathbb{R}^{3}$ with impedance boundary data it is known that the constant in the corresponding bound satisfies the estimate $C=O(k)$ (for example, this can be proved by combining estimate (2) of Theorem 1 of [12] with the argument of Lemma 4.5, involving a function corresponding to $\left.u_{0}\right)$. For a somewhat analogous one-dimensional problem the inf-sup constant is also $O(k)$ as $k \rightarrow \infty$ (Theorem 4.2 of $[19])$.

We proceed now to establish that Lemmas 4.6 and 4.8 hold for much more general boundaries, namely those satisfying (4.1). To establish this we first prove the following technical lemma.

Lemma 4.10. If (4.1) holds, then, for every $\phi \in C_{0}^{\infty}(D)$, there exists $f \in$ $C^{\infty}\left(\mathbb{R}^{n-1}\right)$ such that

$$
\operatorname{supp} \phi \subset D^{\prime}:=\left\{x \in \mathbb{R}^{n}: x_{n}>f(\tilde{x}), \tilde{x} \in \mathbb{R}^{n-1}\right\}
$$

and $U_{f_{+}} \subset D^{\prime} \subset D$.

Proof. Let $S:=\operatorname{supp} \phi \backslash U_{f_{+}}$. Then either $S=\emptyset$, in which case $f(\tilde{x}) \equiv f_{+}$has the properties claimed, or $S \neq \emptyset$.

Thus, suppose $S \neq \emptyset$ and let $\delta:=\operatorname{dist}(S, \partial D) / 2$. Then $\delta>0$ and, defining $G:=\left\{x+s e_{n}: x \in S, s \geq 0\right\}, \operatorname{dist}(G, \partial D)=\operatorname{dist}(S, \partial D)=2 \delta$. Let $G_{\delta}:=\left\{x \in \mathbb{R}^{n}:\right.$ $\operatorname{dist}(x, G)<\delta\}$ and let $A$ and $A_{\delta}$ denote the projections of $G$ and $G_{\delta}$, respectively, onto the $O x_{1} \cdots x_{n-1}$ plane.

Let $N \in \mathbb{N}$ and $S_{j} \subset \mathbb{R}^{n-1}, j=1, \ldots, N$, be such that each $S_{j}$ is measurable and nonempty, $S_{j} \cap S_{m}=\emptyset$ for $j \neq m$,

$$
A_{\delta} \subset \bigcup_{j=1}^{N} S_{j},
$$

and $\operatorname{diam}\left(S_{j}\right) \leq \delta / 2, j=1, \ldots, N$. For $j=1, \ldots, N$ choose $\tilde{x}_{j} \in S_{j}$ and let

$$
f_{j}:=\inf \left\{x_{n} \in \mathbb{R}: x=\left(\tilde{x}_{j}, x_{n}\right) \in G_{\delta} \cup \Gamma_{f_{+}}\right\} .
$$

Then $f_{-} \leq f_{j} \leq f_{+}, j=1, \ldots, N$. Define $\tilde{f}: \mathbb{R}^{n-1} \rightarrow \mathbb{R}$ by

$$
\tilde{f}(\tilde{x}):= \begin{cases}f_{j} & \text { if } \tilde{x} \in S_{j}, j=1, \ldots, N, \\ f_{+} & \text {otherwise. }\end{cases}
$$

Then $\tilde{f} \in L^{\infty}\left(\mathbb{R}^{n-1}\right)$; in fact $\tilde{f}$ is a simple function and $f_{-} \leq \tilde{f}(\tilde{x}) \leq f_{+}, \tilde{x} \in \mathbb{R}^{n-1}$. Choose $\epsilon$ with $0<\epsilon<\delta / 2$ and let $J \in C_{0}^{\infty}\left(\mathbb{R}^{n-1}\right)$ be such that $J \geq 0, J(\tilde{x})=0$ if $|\tilde{x}| \geq \epsilon$, and $\int_{\mathbb{R}^{n-1}} J(\tilde{x}) d \tilde{x}=1$. Define $f \in C^{\infty}\left(\mathbb{R}^{n-1}\right)$ by

$$
f(\tilde{x}):=\int_{\mathbb{R}^{n-1}} J(\tilde{x}-\tilde{y}) \tilde{f}(\tilde{y}) d \tilde{y}, \quad \tilde{x} \in \mathbb{R}^{n-1},
$$

and let $D^{\prime}$ be defined as in the statement of the lemma. Then $f$ and $D^{\prime}$ have the properties claimed. 
To see that this is true note first that

$$
\min _{|\tilde{y}-\tilde{x}|<\epsilon}|\tilde{f}(\tilde{y})| \leq f(\tilde{x}) \leq \max _{|\tilde{y}-\tilde{x}|<\epsilon}|\tilde{f}(\tilde{y})|, \quad \tilde{x} \in \mathbb{R}^{n-1},
$$

so that $U_{f_{+}} \subset D^{\prime}$. If $\tilde{x} \in A$, then $|\tilde{y}-\tilde{x}|<\epsilon$ implies that $\tilde{y} \in A_{\delta}$ and so (4.19) implies that

$$
f(\tilde{x}) \leq \max _{j=1, \ldots, N,\left|\tilde{x}_{j}-\tilde{x}\right|<\epsilon+\delta / 2} f_{j}
$$

so that $f(\tilde{x}) \leq f_{m}$ for some $m$ for which $\left|\tilde{x}_{m}-\tilde{x}\right|<\epsilon+\delta / 2$. Now let $x=\left(\tilde{x}, f_{m}\right)$, $y=\left(\tilde{x}_{m}, f_{m}\right)$. Then $|x-y|=\left|\tilde{x}-\tilde{x}_{m}\right|<\epsilon+\delta / 2$ and $\operatorname{dist}(y, G)=\delta$ so that

$$
\operatorname{dist}(x, G) \geq \operatorname{dist}(y, G)-|x-y| \geq \delta-(\epsilon+\delta / 2)>0 .
$$

Thus $x \notin G$ and so $(\tilde{x}, f(\tilde{x})) \notin G$. Thus $S \subset G \subset D^{\prime}$ and so $\operatorname{supp} \phi \subset U_{f_{+}} \cup S \subset D^{\prime}$.

Arguing similarly, for all $\tilde{x} \in \mathbb{R}^{n-1}$, either $f(\tilde{x})=f_{+}$, in which case $\left(\tilde{x}, x_{n}\right) \in D$ for $x_{n}>f(\tilde{x})$, or $f(\tilde{x}) \geq f_{m}$ for some $m$ for which $\left|\tilde{x}_{m}-\tilde{x}\right|<\epsilon+\delta / 2$. In this latter case, defining $x=\left(\tilde{x}, f_{m}\right)$ and $y=\left(\tilde{x}_{m}, f_{m}\right)$, it holds that

$$
\operatorname{dist}(x, G) \leq \operatorname{dist}(y, G)+|x-y| \leq \delta+\epsilon+\delta / 2<2 \delta
$$

so that $x \in D$ and hence $(\tilde{x}, f(\tilde{x})) \in D$. Thus, for all $\tilde{x} \in \mathbb{R}^{n-1},\left(\tilde{x}, x_{n}\right) \in D$ for $x_{n}>f(\tilde{x})$, i.e., $D^{\prime} \subset D$.

With this preliminary lemma we can proceed to show that Lemma 4.6 holds whenever (4.1) holds.

Lemma 4.11. Suppose (4.1) holds, $H \geq f_{+}, g \in L^{2}\left(S_{H}\right)$, and $w \in V_{H}$ satisfies

$$
b(w, \phi)=-(g, \phi) \quad \text { for all } \phi \in V_{H} .
$$

Then

$$
\|w\|_{V_{H}} \leq k^{-1} \tilde{C}\|g\|_{2}
$$

where $\tilde{C}=\frac{\kappa}{\sqrt{2}}(\kappa+1)^{2}$.

Proof. Let $\tilde{V}:=\left\{\left.\phi\right|_{S_{H}}: \phi \in C_{0}^{\infty}(D)\right\}$. Then $\tilde{V}$ is dense in $V_{H}$. Suppose $w$ satisfies (4.20) and choose a sequence $\left(w_{m}\right) \subset \tilde{V}$ such that $\left\|w_{m}-w\right\|_{V_{H}} \rightarrow 0$ as $m \rightarrow \infty$. Then $w_{m}=\left.\phi_{m}\right|_{S_{H}}$, with $\phi_{m} \in C_{0}^{\infty}(D)$, and, by Lemma 4.10, there exists $f_{m} \in C^{\infty}\left(\mathbb{R}^{n-1}\right)$ such that supp $\phi_{m} \subset D_{m}$ and $U_{f_{+}} \subset D_{m} \subset D$, where $D_{m}:=\{x \in$ $\left.\mathbb{R}^{n}: x_{n}>f_{m}(\tilde{x}), \tilde{x} \in \mathbb{R}^{n-1}\right\}$. Let $V_{H}^{(m)}$ and $b_{m}$ denote the space and sesquilinear form corresponding to the domain $D_{m}$. That is, where $S_{H}^{(m)}:=D_{m} \backslash \overline{U_{H}}, V_{H}^{(m)}$ is defined by $V_{H}^{(m)}:=\left\{\left.\phi\right|_{S_{H}^{(m)}}: \phi \in H_{0}^{1}\left(D_{m}\right)\right\}$ and $b_{m}$ is given by (2.22) with $S_{H}$ and $V_{H}$ replaced by $S_{H}^{(m)}$ and $V_{H}^{(m)}$, respectively. Then $S_{H}^{(m)} \subset S_{H}$ and, if $v_{m} \in V_{H}^{(m)}$ and $v$ denotes $v_{m}$ extended by zero from $S_{H}^{(m)}$ to $S_{H}$, it holds that $v \in V_{H}$. Via this extension by zero, we can regard $V_{H}^{(m)}$ as a subspace of $V_{H}$ and regard $w_{m}$ as an element of $V_{H}^{(m)}$.

For all $v \in V_{H}^{(m)} \subset V_{H}$, we have

$$
b_{m}\left(w_{m}, v\right)=b\left(w_{m}, v\right)=-(g, v)-b\left(w-w_{m}, v\right) .
$$


By Lemma 4.8 , there exist unique $w_{m}^{\prime}, w_{m}^{\prime \prime} \in V_{H}^{(m)}$ such that

$$
b_{m}\left(w_{m}^{\prime}, v\right)=-(g, v), \quad v \in V_{H}^{(m)},
$$

and

$$
b_{m}\left(w_{m}^{\prime \prime}, v\right)=-b\left(w-w_{m}, v\right), \quad v \in V_{H}^{(m)} .
$$

Clearly $w_{m}=w_{m}^{\prime}+w_{m}^{\prime \prime}$ and, by Lemma 4.6 ,

$$
\left\|w_{m}^{\prime}\right\|_{V_{H}^{(m)}} \leq k^{-1} \tilde{C}\|g\|_{2}
$$

while, by Lemmas 4.8 and 3.4,

$$
\left\|w_{m}^{\prime \prime}\right\|_{V_{H}^{(m)}} \leq 2 C\left\|w-w_{m}\right\|_{V_{H}} .
$$

Thus

$$
\|w\|_{V_{H}}=\lim _{m \rightarrow \infty}\left\|w_{m}\right\|_{V_{H}^{(m)}} \leq k^{-1} \tilde{C}\|g\|_{2} .
$$

Theorem 4.1 now follows by combining Lemmas 4.11, 4.5, and 4.4 with Corollary 4.3 .

Acknowledgments. The authors are grateful to Andreas Kirsch and Martin Thomas for helpful comments on an earlier version of this paper.

\section{REFERENCES}

[1] R. A. Adams, Sobolev Spaces, Academic Press, New York, 1975.

[2] H. D. Alber, A quasi-periodic boundary value problem for the Laplacian and the continuation of its resolvent, Proc. Roy. Soc. Edinburgh, 82A (1979), pp. 251-272.

[3] T. Arens, S. N. Chandler-Wilde, and K. Haseloh, Solvability and spectral properties of integral equations on the real line: I. Weighted spaces of continuous functions, J. Math. Anal. Appl., 272 (2002), pp. 276-302.

[4] T. Arens, S. N. Chandler-Wilde, and K. Haseloh, Solvability and spectral properties of integral equations on the real line: II. $L^{p}$ spaces and applications, J. Integral Equations Appl., 15 (2003), pp. 1-35.

[5] T. AREns And T. Hohage, On radiation conditions for rough surface scattering problems, IMA J. Appl. Math., to appear; also available online from http://imamat.oupjournals.org/cgi/ content/abstract/hxh065v1.

[6] G. Bao, D. Dobson, And J. Cox, Mathematical studies in rigorous grating theory, J. Opt. Soc. Amer. A, 12 (1995), pp. 1029-1042.

[7] M. Cadilhac, Some mathematical aspects of the grating theory, in Electromagnetic Theory of Gratings, R. Petit, ed., Springer, Berlin, 1980, pp. 53-62.

[8] S. N. Chandler-Wilde, E. Heinemeyer, and R. Potthast, Acoustic scattering by mildly rough unbounded surfaces in three dimensions, submitted.

[9] S. N. Chandler-Wilde, C. R. Ross, And B. Zhang, Scattering by rough surfaces, in Proceedings of the Fourth International Conference on Mathematical and Numerical Aspects of Wave Propagation, J. DeSanto, ed., SIAM, Philadelphia, 1998, pp. 164-168.

[10] S. N. Chandler-Wilde, C. R. Ross, And B. Zhang, Scattering by infinite one-dimensional rough surfaces, Proc. R. Soc. Lond. Ser. A, 455 (1999), pp. 3767-3787.

[11] S. N. Chandler-Wilde And B. Zhang, A uniqueness result for scattering by infinite rough surfaces, SIAM J. Appl. Math., 58 (1998), pp. 1774-1790.

[12] P. Cummings And X. Feng, Sharp regularity coefficient estimates for complex-valued acoustic and elastic Helmholtz equations, Math. Models Methods Appl. Sci., to appear.

[13] J. A. De Santo, Scattering by rough surfaces, in Scattering: Scattering and Inverse Scattering in Pure and Applied Science, R. Pike and P. Sabatier, eds., Academic Press, New York, 2002, pp. 15-36. 
[14] D. Eidus AND A. Vinnik, On radiation conditions for domains with infinite boundaries, Soviet Math. Dokl., 15 (1974), pp. 12-15.

[15] J. Elschner And G. Schmidt, Diffraction in periodic structures and optimal design of binary gratings I: Direct problems and gradient formulas, Math. Methods Appl. Sci., 81 (1998), pp. 1307-1328.

[16] J. Elschner and M. Yamamoto, An inverse problem in periodic diffractive optics: Reconstruction of Lipschitz grating profiles, Appl. Anal., 81 (2002), pp. 1307-1328.

[17] D. Gilbarg and N. S. Trudinger, Elliptic Partial Differential Equations of Second Order, 2nd ed., Springer, Berlin, 1983.

[18] V. Y. GotLiB, Solutions of the Helmholtz equation, concentrated near a plane periodic boundary, J. Math. Sci., 102 (2000), pp. 4188-4194.

[19] F. Inlenburg, Finite Element Analysis of Acoustic Scattering, Appl. Math. Sci. 132, Springer, Berlin, 1998.

[20] A. Kirsch, Diffraction by periodic structures, in Inverse Problems in Mathematical Physics, L. Pävärinta and E. Somersalo, eds., Springer, Berlin, 1993, pp. 87-102.

[21] J. Melenk, On Generalized Finite Element Methods, Ph.D. thesis, University of Maryland, College Park, MD, 1995.

[22] V. MinskiI, Sommerfeld radiation conditions for second-order differential operator in a domain with infinite border, J. Differential Equations, 48 (1983), pp. 157-176.

[23] J. A. OGilvy, Theory of Wave Scattering from Random Rough Surfaces, Adam Hilger, Bristol, 1991.

[24] M. Saillard and A. Sentenac, Rigorous solutions for electromagnetic scattering from rough surfaces, Waves Random Media, 11 (2001), pp. R103-R137.

[25] V. Vogelsang, Das Ausstrahlungsproblem fur elliptische Differentialgleichungen in Gebieten mit unbeschranktem Rand, Math. Z., 144 (1975), pp. 101-124.

[26] A. G. Voronovich, Wave Scattering from Rough Surfaces, Springer, Berlin, 1994.

[27] K. WARnick AND W. C. CheW, Numerical simulation methods for rough surface scattering, Waves Random Media, 11 (2001), pp. R1-R30.

[28] B. Zhang, An integral equation method for scattering by an infinite Lipschitz surface, in Proc. JEE '02, European Symp. Numerical Methods in Electromagnetics, Toulouse, 2002, pp. 31-35.

[29] B. Zhang and S. N. Chandler-Wilde, Acoustic scattering by an inhomogeneous layer on a rigid plate, SIAM J. Appl. Math., 58 (1998), pp. 1931-1950.

[30] B. Zhang And S. N. Chandler-Wilde, Integral equation methods for scattering by infinite rough surfaces, Math. Methods Appl. Sci., 26 (2003), pp. 463-488. 